\title{
Dynamics of a Charge Qubit Encoded in a Double Quantum Dot
}

\author{
J. ŁUCZAK*, B.R. BuŁKA \\ Institute of Molecular Physics, Polish Academy of Sciences, Mariana Smoluchowskiego 17, 60-179 Poznań, Poland
}

\begin{abstract}
We study the short time dynamics of a charge qubit encoded in two coherently coupled quantum dots connected to external electrodes. In the regime of sequential tunneling the waiting time distribution is used to investigate in details statistics of the tunneling events and their correlations. We show that the coherent rotation of the qubit state on the Bloch sphere can be seen in tunneling events when an electron is injected and leave the system. Decoherence, relaxation of the qubit state as well as leakage processes are studied by means of transient currents.
\end{abstract}

DOI: 10.12693/APhysPolA.133.748

PACS/topics: 03.67.Lx 73.63.Kv 73.23.Ra

\section{Introduction}

Quantum computation requires qubits which can be encoded, controlled and read-out in a very short time scale. Nowadays, qubits based on quantum dots (QDs) are very promising due to great progress in fabrication of systems of many coherently coupled QD which can act as a quantum register. Qubits can be encoded in a single [1], in two [2] as well as in three electron states [3]. One of the simplest setup, in which a qubit can by realized, is a double quantum dot (DQD) system with a single electron [1]. The charge degrees of freedom allows to encode the qubit with a long coherence time and a gate operation time in a picosecond time scale [4]. In this case the qubit states, which corresponds to the north and the south pole of the Bloch sphere, are set by position of the electron in the double well potential. The control of the qubit states can be performed by applying voltage pulses to a drain contact of the DQD [5] or by detuning between local site energies [1], whereas the read-out by a noninvasive quantum point contact QPC detector [1]. In this paper we investigate the charge qubit encoded in a single electron in the DQD system connected to external electrodes. The main idea is to use the transient transport to study the coherent qubit dynamics and the read-out process in a short time scale. We use the waiting time distribution [6] to investigate in details statistics of tunneling events when an electron is injected and leave the DQD. Coupling with the external electrodes can cause decoherence processes which destroy the information encoded in the qubit. Therefore, we study transient currents flowing through the system to find characteristic times for a relaxation, a decoherence and leakage processes.

\section{Model of charge qubit}

We consider a system of two coherently coupled quantum dots (DQD) in which one of the dots is connected to

\footnotetext{
* corresponding author; e-mail jakub.luczak@ifmpan.poznan.pl
}

the source (left) and drain (right) electrodes (see insert in Fig. 1). The isolated DQD is described by the Hamiltonian

$$
H=\sum_{i} \epsilon_{i} c_{i}^{\dagger} c_{i}+t_{i j}\left(c_{i}^{\dagger} c_{j}+c_{j}^{\dagger} c_{i}\right),
$$

where $\epsilon_{i}$ is a local energy at the dot $i, t$ is a hopping parameter and $c_{i}^{\dagger}\left(c_{i}\right)$ denotes a creation (annihilation) operator of an electron. We assume strong Coulomb interactions which means that in the system can be only one electron. The charge qubit states are defined as $|10\rangle$ and $|01\rangle$, with the electron on the first and the second dot, respectively. They correspond the qubit oriented toward the north and the south pole on the Bloch sphere. There is also the leakage state $|00\rangle$ — without any electron in the system.

The dynamics of the system is govern by the master equation $\dot{\rho}=-\mathrm{i}[H, \rho]+D(\rho)$, where $D(\rho)$ is the Lindblad dissipator [7] describing connections with the electrodes. For the considered model it is convenient to express the density matrix as $\rho=$ $\left\{\rho_{10,10}, \rho_{01,01}, \rho_{10,01}, \rho_{01,10}, \rho_{00,00}\right\}^{T}$ and rewrite the Master equation in the form $\dot{\rho}=\mathcal{L} \rho$ with the Liouvillian operator

$$
\mathcal{L}=\left[\begin{array}{ccccc}
-\Gamma^{-} & 0 & \mathrm{i} t & -\mathrm{i} t & \Gamma^{+} \\
0 & 0 & -\mathrm{i} t & \mathrm{i} t & 0 \\
\mathrm{i} t & -\mathrm{i} t & -\frac{\Gamma^{-}}{2}-\Delta \epsilon & 0 & 0 \\
-\mathrm{i} t & \mathrm{i} t & 0 & -\frac{\Gamma^{-}}{2}+\Delta \epsilon & 0 \\
\Gamma^{-} & 0 & 0 & 0 & -\Gamma^{+}
\end{array}\right] .
$$

Here, $\Delta \epsilon=\epsilon_{1}-\epsilon_{2}$ is the detuning parameter of the local energy levels and $\Gamma^{ \pm}=\Gamma^{L \pm}+\Gamma^{R \pm}$. The transfer rates from the left $(\mathrm{L})$ and right $(\mathrm{R})$ electrode to the first dot of the DQD system are given by the Fermi golden rule: $\Gamma^{L(R) \pm}=\gamma_{L(R)} f^{ \pm}\left(\epsilon_{1}-\mu_{L(R)}\right)$, where $\gamma_{L(R)}$ is a parameter characterizing tunneling probability through the barrier $L(R), f^{+}$denotes the Fermi distribution function, $f^{-}=1-f^{+}$and $\mu_{L(R)}$ the electrochemical potentials in the left and the right electrode.

Our studies focus on short time dynamics of the charge qubit described by the Hamiltonian (1) with a single electron. The qubit dynamics is studied first by the waiting 
time distribution and next by means of the transient currents.

\section{Analysis of waiting time distribution}

The waiting time distribution (WTD) is a probability for the delay time between two subsequent jumps processes $k$ and $l$ when the system is initially in the stationary state $\rho_{0}[6]$

$$
w_{k l}=\frac{\operatorname{Tr}\left[\mathcal{J}_{l} \mathrm{e}^{\mathcal{L}_{0} \tau} \mathcal{J}_{k} \rho_{0}\right]}{I_{l}},
$$

where $\mathcal{J}_{l}$ is the jump operator contains off-diagonal elements of the Liouvillian (2) which correspond to the tunneling processes through the barrier $l . I_{l}=\operatorname{Tr}\left[\mathcal{J}_{l} \rho_{0}\right]$ is a stationary current due to this jump process with the density matrix $\rho_{0}$ given by $\mathcal{L} \rho_{0}=0$. For $k \neq l$ the Liouvillian $\mathcal{L}_{0}=\mathcal{L}-\mathcal{J}_{k}-\mathcal{J}_{l}$ describes the evolution of the system without the jump processes $l$ and $k$.

Figure 1 presents $w_{L R}$ (black curve), which corresponds the WTD for the occupation time of an electron at the first jump to the drain electrode. The red curve shows $w_{R L}$ for the distribution of the waiting time in the empty state to the first jump from the source electrode. At the initial time $\tau=0$, the system is in the stationary state for which all three states are occupied with probability $1 / 3$, therefore $w_{L R}(\tau=0)=w_{R L}(\tau=0)$. One can see that $w_{R L}$ (red curve) decreases with time monotonously and is given by the expression $w_{R L}=\Gamma^{L+} \mathrm{e}^{\left(-\Gamma^{L+} \tau\right)}$. The situation is different for $w_{L R}$ which exhibits oscillatory behaviour. First the system is charged by the jump process $\mathcal{J}_{L}$ from the $L$ electrode. The qubit is encoded in the state $|10\rangle$ corresponding to the north pole of the Bloch sphere. Next one can observe the coherent rotation of the qubit state which evolution is described by $\mathrm{e}^{\left(\mathcal{L}-\mathcal{J}_{L}-\mathcal{J}_{R}\right) \tau}$. If the electron occupies the second dot, the qubit points the south pole of the Bloch sphere, then the jump process $J_{R}$ cannot occur and $w_{L R}=0$. In this case the transport through the system is blocked until the electron returns to the first QD. The waiting time distribution $w_{L R}$ is given by (for $\Delta \epsilon=0)$

$$
\begin{gathered}
w_{L R}=\frac{\Gamma^{R-}}{\phi^{2}} \mathrm{e}^{-\frac{\Gamma^{R-\tau}}{2}}\left\{8 t^{2}\left[1+\cos \left(\frac{\phi \tau}{2}\right)\right]\right. \\
\left.-\Gamma^{R-}\left[\Gamma^{R-} \cos \left(\frac{\phi \tau}{2}\right)+\phi \sin \left(\frac{\phi \tau}{2}\right)\right]\right\},
\end{gathered}
$$

where the period $\phi=\sqrt{16 t^{2}-\left(\Gamma^{R-}\right)^{2}}$.

\section{Analysis of transient currents}

The studies of the waiting time distribution tells about delay time between subsequence jump processes. However to investigate the qubit dynamics in more details one needs to analyse relaxation and decoherence times. Here we consider transient currents which can be used to read-out of the qubit state and give information about dynamics as well as relaxation, decoherence and leakage processes. The transient currents are defined as

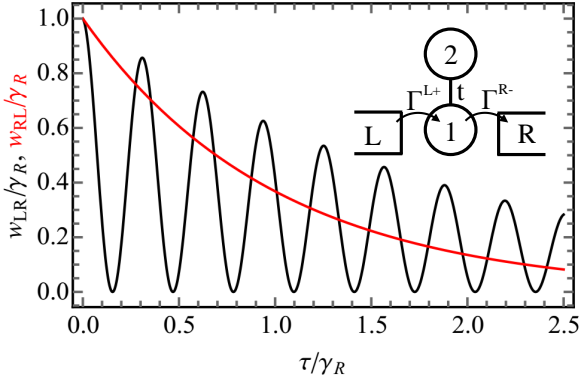

Fig. 1. The waiting time distribution $w_{L R}$ - black curve and $w_{R L}$ - red curve for $\Delta \epsilon=0, t=1, \gamma_{L}=$ $\gamma_{R}=0.1, k T=0.01$. Insert shows the model of the system..

$$
\begin{aligned}
& I_{L}(\tau)=e\left[\Gamma^{L+} \rho_{00,00}(\tau)-\Gamma^{L-} \rho_{10,10}(\tau)\right], \\
& I_{R}(\tau)=e\left[\Gamma^{R-} \rho_{10,10}(\tau)-\Gamma^{R+} \rho_{00,00}(\tau)\right],
\end{aligned}
$$

for the left and the right tunnel junction, respectively. Notice, that in general $I_{L}(\tau) \neq I_{R}(\tau)$ due to timedependent charge accumulation in DQD. The both currents are equal only in the stationary limit for $\tau \rightarrow \infty$.

In order to have better insight into dynamics of the qubit we use the Bloch base $s_{z}=\rho_{10,10}-\rho_{01,01}, s_{x}=$ $\rho_{10,01}+\rho_{01,10}$ and $s_{y}=i\left(\rho_{10,01}-\rho_{01,10}\right)$. The equation of motion for the qubit is given by

$$
\begin{gathered}
\dot{s}_{x}=-\frac{1}{2} \Gamma^{R-} s_{x}+\Delta \epsilon s_{y}, \\
\dot{s}_{y}=-\frac{1}{2} \Gamma^{R-} s_{y}-\Delta \epsilon s_{x}-2 t s_{z}, \\
\dot{s}_{z}=-\frac{1}{2} \Gamma^{R-} s_{z}+2 t s_{y}-\left(\Gamma^{L+}+\frac{1}{2} \Gamma^{R-}\right) \rho_{00,00}+\frac{1}{2} \Gamma^{R+}, \\
\dot{\rho}_{00,00}=-\left(\Gamma^{L+}+\frac{1}{2} \Gamma_{R-}\right) \rho_{00,00}-\frac{1}{2} \Gamma^{R-} s_{z}+\frac{1}{2} \Gamma^{R-} .
\end{gathered}
$$

These equations are similar to the optical Bloch equations and therefore, by analogy, one can extract the relaxation rate $1 / T_{1} \approx \Gamma^{R-} / 2$ and the decoherence rate $1 / T_{2} \approx \Gamma^{R-} / 2$. Notice that in the optical Bloch equation $T_{2}=2 T_{1}$, but here $T_{2}=T_{1}$ because the thermal transitions between the qubits states are neglected and we consider the relaxation processes only. The time $T_{2}$ describes how fast the coherence between qubit states is lost due to interaction with the electrodes. The relaxation time $T_{1}$ gives information about the evolution of the $z$ component of the pseudospin. These equations describe the qubit rotation in the presence of a pseudo magnetic field $B=(2 t, 0, \Delta \epsilon)$. The last equation describes a leakage process from the qubit subspace to the state $|00\rangle$, which causes the collapse of the Bloch sphere. One can estimate the leakage time as $1 / T_{\text {leak }} \approx \Gamma^{L+}+\Gamma^{R-} / 2$.

Figure 2 presents the transient currents $I_{L}(\tau)$ (red curve) and $I_{R}(\tau)$ (black curve) for the initial state $|10\rangle$. For low temperature and unidirectional transport, $I_{R}(\tau)$ is proportional to the occupation probability $\rho_{10,10}(\tau)$. It means, that the qubit states can be read-out by measurement of the transient currents. The oscillatory behavior of $I_{R}(\tau)$ is similar to $w_{L R}$ in Fig. 1, because the both quantities give information about the dynamics of 


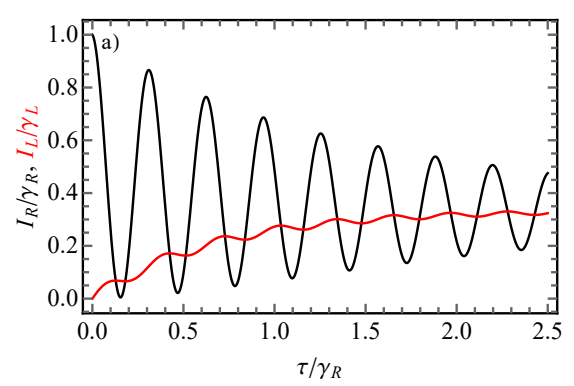

Fig. 2. Time dependence of the transient current $I_{L}$ (black curve) and $I_{R}$ (red curve) flowing through the left and right tunnel junction, respectively, for the qubit encoded in the state $|10\rangle$ at $\tau=0$. The parameters are the same as in Fig. 1. b) Rotation of the qubit on the Bloch sphere. The black vector corresponds to the initial state, whereas the green one to the final state. Dumping is due to relaxation and leakage processes with rates $1 / T_{1}$ and $1 / T_{\text {leak }}$, respectively.

the qubit state and its coherent rotation on the Bloch sphere. The minimum value of $I_{R}(\tau)$ is reached when the qubit state becomes oriented to the south pole and the maximum is for the orientation to the north pole. Moreover $I_{R}(\tau)$ shows also the relaxation to the stationary state with time $T_{1}$. The red curve shows the transient current $I_{L}(\tau)$ flowing through the left tunnel junction which is proportional to the state $\rho_{00,00}(\tau)$. At the initial time the current is zero and then increases with the ratio $1 / T_{\text {leak }}$ which is related to the leakage processes out of the qubit subspace. For longer times the current relax to the stationary state with $1 / T_{1}$. One can see that current measurements give information about the relaxation and the leakage processes which cannot be obtained from WTD analysis.

The bottom panel in Fig. 2 shows the rotation of the pseudospin vector of the qubit on the Bloch sphere. The black vector corresponds to the initial $\rho_{10,10}(\tau)$ state which is directed to the north pole on the Bloch sphere, and the final state is represented by the green arrow. The vector rotates around a pseudo magnetic field $B=(2 t, 0, \Delta \epsilon)$ with the radius which is diminished in time due to decoherence rate $1 / T_{1}$. The leakage process manifest itself in the short time scale in a few first cycles.

\section{Conclusion}

We considered the dynamics of the charge qubit encoded in two coherently coupled quantum dots. The system was weakly coupled to the external electrodes by tunnel junctions and its dynamics was assumed to be Markovian. Two complementary methods were used: the waiting time distribution (WTD) and the transient currents. In particular the mutual correlations between jump events when an electron is injected and leave the DQD show oscillatory behaviour which corresponds to the coherent rotation of the qubit state on the Bloch sphere induced by a pseudo-magnetic field $B=(2 t, 0, \Delta \epsilon)$. Contact with the external electrodes leads to decoherence and relaxation processes which affect the qubit evolution. These processes were analysed by the transient currents, which showed oscillations due to coherent evolution and the characteristic decoherence and relaxation times (inversely proportional to the tunneling rate to the drain electrode). Moreover, we showed that the leakage process to the non-qubit state $|00\rangle$ manifest itself in a short time scale.

\section{Acknowledgments}

The research was financed by National Science Centre, Poland - project number 2016/21/B/ST3/02160.

\section{References}

[1] K.D. Petersson, J.R. Petta, H. Lu, A.C. Grossard, Phys. Rev. Lett. 105, 246804 (2010).

[2] V. Srinivasa, J.M. Taylor, Phys. Rev. B 92, 235301 (2015).

[3] L. Gaudreau, G. Granger, A. Kam, G.C. Aers, S.A. Studenikin, P. Zawadzki, M. Pioro-Ladriere, Z.R. Wasilewski, A.S. Sachrajda, Nature Phys. 8, 54 (2012).

[4] D. Vion, A. Aassime, A. Cottet, P. Joyez, H. Pothier, C. Urbina, D. Esteve, M.H. Devoret, Science 296, 886 (2002).

[5] G. Shinkai, T. Hayashi, T. Ota, T. Fujisawa, Phys. Rev. Lett. 103, 056802 (2009).

[6] T. Brandes, Ann. Phys. (Berlin) 17, 477 (2008).

[7] M. Busl, G. Platero, Phys. Rev. B 82, 205304 (2010). 